\title{
Diagnostic Value of 3D FIESTA and 3D COSMIC Sequences in Imaging of Lumbar Radiculopathy
}

\author{
Abubacker Sulaiman $\mathrm{F}^{1}$, Amritha $\mathrm{A}^{2}$, Farooque $\mathrm{MC}^{3}$, Ashraf Ahmed B ${ }^{4}$, Praveen $\mathrm{S}^{5}$, Vijay Prabhu $\mathrm{R}^{6}$ \\ ${ }^{1}$ Professor, Department of Radiology, Chettinad Hospital \& Research Institute, ${ }^{2}$ Final Year Postgraduate, Department of \\ Radiology, Chettinad Hospital \& Research Institute, ${ }^{3}$ Final Year Postgraduate, Department of Radiology, Chettinad Hospital \& \\ Research Institute, ${ }^{4}$ Associate Professor, Department of Radiology, Chettinad Hospital \& Research Institute, ${ }^{5}$ Senior Resident, \\ Department of Radiology, Chettinad Hospital \& Research Institute, ${ }^{6}$ Senior Resident, Department of Radiology, Chettinad \\ Hospital \& Research Institute, Kelambakkam, Tamil Nadu, India
}

Corresponding author: Dr. Amritha A, Final Year Postgraduate, Department of radiology, Chettinad Hospital \& Research Institute, Rajiv Gandhi Salai, Kelambakkam, Chennai, Tamilnadu, India

DOI: http://dx.doi.org/10.21276/ijcmsr.2019.4.4.11

How to cite this article: Abubacker Sulaiman F, Amritha A, Farooque MC, Ashraf Ahmed B, Praveen S, Vijay Prabhu R. Diagnostic value of 3D FIESTA and 3D COSMIC sequences in imaging of lumbar radiculopathy. International Journal of Contemporary Medicine Surgery and Radiology. 2019;4(4):D47-D50.

\section{A B S T R A C T}

Introduction: The diagnosis of nerve root compression and spinal canal stenosis by normal 2D lumbar vertebra MRI can be difficult. Through MRI, the diagnosis of nerve root compression and spinal canal stenosis by normal 2D lumbar vertebra is difficult sometimes. Which becomes challenging to find out the level responsible for certain conditions, such as epidural radiculopathy. Therefore, the aim of the present study was to evaluate whether the 3D sequences had any superiority over conventional 2D sequences in the evaluation of lumbar radiculopathy.

Material and methods: The present research was a cross-sectional prospective study, which was, conducted on two groups of patients who were separately examined and two radiologists did further evaluation. There were 15 control patients and 58 experimental patients. About 58 patients with clinical suspicion of lumbar radiculopathy referred to Department of Radiology for imaging were included in this study. The 3D balanced sequences acquired were FIESTA and COSMIC.

Results: In the present study, it was found that between T2 weighted, 3D Fiesta and 3D Cosmic sequences showed significant statistical difference between these three sequences. Significant difference $(p=0.035)$ between T2 weighted and 3D FIESTA (fast imaging employing steady state acquisition) was noted. Similarly, 3D COSMIC (coherent oscillatory state acquisition for the manipulation of image contrast) and T2 weighted sequence shows significant difference at $p$ value 0.001

Conclusion: The findings from the present study suggest that 3D sequences are better than 2D sequences in the imaging of lumbar radiculopathy and also 3D COSMIC definitely has advantages over 3D FIESTA sequence.

Keywords: Magnetic Resonance Imaging; Three-dimensional; Intervertebral Foramen; Lumbar Nerve Root, 3D Fiesta, 3DCosmic

\section{INTRODUCTION}

Low-back pain (LBP) has become one of the most common ailments these days. Various causes could lead to low back pain which could further be categorized into mechanical, non-mechanical and visceral causes. Commonly encountered reasons for LBP are degenerative disc disease, spondylolysis, spondylolisthesis, and spinal stenosis. MRI has become the method of choice for radiological evaluation of the spine. MRI has greater sensitivity in detecting various anatomic changes that could lead to radiculopathy. Recent advances like $3 \mathrm{D}$ sequences can be used for acquiring further information using thin slices with multi-planar reconstruction. ${ }^{1}$

Lumbar radiculopathy (LR) is a pain syndrome caused by the compression or irritation of nerve roots in the lower back. Low back pain (LBP) with a prevalence of up to $84 \%$ is the most common musculoskeletal condition affecting the adult population. Many authors suggested the definition of chronic pain as the pain in the lower back (CLBP) which lasts beyond the expected period of healing, avoiding this close time criterion. This definition is very important, as it underlines the concept that chronic pain has well-defined underlying pathological causes and that it is a disease, not a symptom and the pain lasting for at least 12 weeks. CLBP is a major welfare and economic problem which represents the leading cause of disability globally. Given this complexity, the diagnostic evaluation of patients with LBP can be quite challenging and needs proper clinical decision-making. ${ }^{2,3}$

Based on its site of occurrence, lumbar radiculopathy is classified into different zones as lateral recess, intervertebral foramen, and external intervertebral foramen zones. Previous studies regarding diagnostic imaging techniques to locate external intervertebral foramen lesions, which are known as far-out-syndrome, has been reported till now. However, because it can be difficult to diagnose these lesions by conventional magnetic resonance imaging (MRI), a more 
specific and accurate imaging technique is the need of the time. $^{4}$

The most used common spinal MRI sequences are two dimensional (2D) i.e.T1- and T2-weighted sagittal followed by axial planes. With T2-weighted images, cerebrospinal fluid (CSF) exhibits higher signals, and the intra-dural nerves exhibit lower signals. To acquire detailed morphologic data three-dimensional (3D) MRI can be used as the settings can be adjusted to obtain thin slices with a high signal-to-noise ratio (SNR), and multiplanar reconstruction (MPR) allows images to be created from the original plane in either the coronal, sagittal, or oblique plane.

It therefore enables observations from multiple directions. Recently, a new technique known as 3D fast-spin echo, has also been found to be effective for rendering nerve roots at high resolution, but the long acquisition time required for the imaging technique is a problem when patients are experiencing pain. ${ }^{5,6}$

Furthermore, with multiple echo collection, the gradientecho sequence improves the T2 contrast between nerve roots and intervertebral foramen stenosis. Over a short acquisition time, this 3D balanced sequence renders the lumbar nerve roots with 3D collection performed. Fast imaging employing steady state acquisition (FIESTA) is a 3D sequence with high fluid sensitivity which enables the high resolution of small intracranial structures and joints by providing exquisite contrast ideally equated for visualization of the internal auditory canal which is ideally suited for T2 imaging through the cervical spine region. ${ }^{7}$ The aim of the study was to assess whether the $3 \mathrm{D}$ sequences were namely 3D FIESTA and 3D COSMIC had any superiority over conventional 2D sequences and also to determine the ideal sequence for the visualization of lumbar spinal root at the level of intervertebral foramen and also for the study of traversing and exiting nerve roots.

\section{MATERIAL AND METHODS}

The present study was a cross-sectional prospective study which was conducted at Chettinad Hospital and Research Institute. The two groups of patients were separately examined and further evaluation was done by two radiologists. There were 15 control patients and 58 experimental patients. About 58 patients with clinical suspicion of lumbar radiculopathy referred to Department of Radiology for imaging were included in this study. The lumbar spines of 15 healthy volunteers ( 7 male and 8 female), in the age group of 24 - 38 years were taken in the present study. The visibility of structures in lumbar spine was scored using a 5 point confident scale. The lumbar spines of 58 patients out of which 28 were males and 30 were female patients, in the age group of 20 to 58 years were examined to be included in this study by using simple random sampling technique.

The 3D balanced sequences acquired were FIESTA and COSMIC. COSMIC can be used with or without fat suppression (FS). The machine used was a Signa HDxt 1.5T (GE Healthcare, Milwaukee, WI, USA) with a spine coil. Ziostation 2 (Ziosoft Inc., Tokyo, Japan) follows the right L5 nerve root and was used to create a MPR. Through areas perpendicular to the L5 nerve root in the intervertebral foramen entrance, intervertebral foramen, and external zones, the nerve root and surrounding tissue signal intensities were measured. In the intervertebral foramen entrance zone followed by the pedicle in the intervertebral foramen zone, and the fat in the intervertebral foramen external zone, the tissue or fluid adjacent to the nerve root was found to be CSF (Figure 1). The results were compared by use of FIESTA, COSMIC and a T2W FSE sequence in terms of contrastto-noise ratio (CNR) with the nerve root was calculated. The CNR was calculated from the following formula ${ }^{8}$

$\mathrm{CNR}=\frac{\mathrm{ISI} \text { (root) }-\mathrm{SI} \text { (peripheral)I }}{\mathrm{SD} \text { (peripheral) }}$

Where SI=Signal intensity; $\mathrm{SD}=\mathrm{Standard}$ deviation

\section{STATISTICAL ANALYSIS}

The data was entered in to the excel sheet and was analyzed with the help of SPSS software version 22. The data was presented in the form of tables and graphs. The Wilcoxon signed ranked test was applied to test the significance between the two parameters. A p value of 0.05 was considered to be statistically significant.

\section{RESULTS}

The maximum visibility score ranged from 1 to 5 in which the maximum score was found to be 5 which means excellent visibility and the minimum was found to be 1 which means not visible. In the present study, it was found that

\begin{tabular}{|l|c|}
\hline Category & Grading \\
\hline Not visible & 1 \\
\hline Barely visible & 2 \\
\hline Adequately visible & 3 \\
\hline Good visibility & 4 \\
\hline Excellent visibility & 5 \\
\hline $\begin{array}{c}\text { Table-1: Shows the distribution of data based on visibility of } \\
\text { structures in lumbar spine among the study subjects }\end{array}$ \\
\hline
\end{tabular}

\begin{tabular}{|l|c|}
\hline Category & Grading \\
\hline Abutting & 0 \\
\hline Indenting & 1 \\
\hline Impingement & 2 \\
\hline Compression & 3 \\
\hline \multicolumn{2}{|c|}{$\begin{array}{c}\text { Table-2: Shows the distribution of data based on degree of } \\
\text { nerve root involvement among the study subjects }\end{array}$} \\
\hline
\end{tabular}

\begin{tabular}{|l|c|c|c|}
\hline Parameters & T2W FSE & Fiesta & Cosmic \\
\hline Mode of acquisition & 2D & 3D & 3D \\
\hline Plane & Sagittal & Coronal & Coronal \\
\hline FOV $(\mathrm{mm})$ & 28 & 28 & 30 \\
\hline TR $(\mathrm{ms})$ & 4320 & 4.9 & 5.1 \\
\hline TE & 11 & 1.9 & 2.5 \\
\hline Matrix & $385 * 256$ & $320 * 256$ & $320 * 320$ \\
\hline Slice thickness $(\mathrm{mm})$ & 3 & 1 & 1 \\
\hline Inter-slice spacing $(\mathrm{mm})$ & 0.5 & - & - \\
\hline P value & 0.086 & 0.009 & 0.001 \\
\hline
\end{tabular}

Table-3: Shows the distribution of data based on image processing and acquisition among the study subjects 


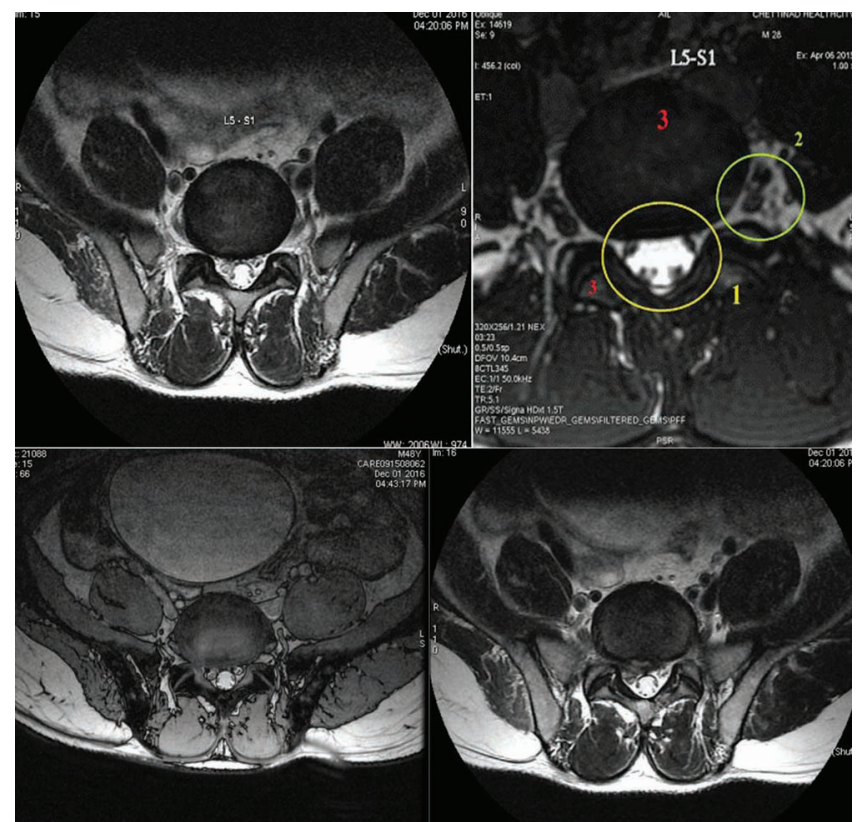

Figure-1: Shows the 3D COSMIC and 3D FIESTA sequence done suggestive of lumbar radiculopathy (1-3 shows Axial T2 weighted image of a 33 year old male patient at the level of L5-S1 level showing loss of normal posterior concavity and facetal joint arthropathy with narrowing of left neural foramina. The nerve root at the level of the neural foramina is not clear).

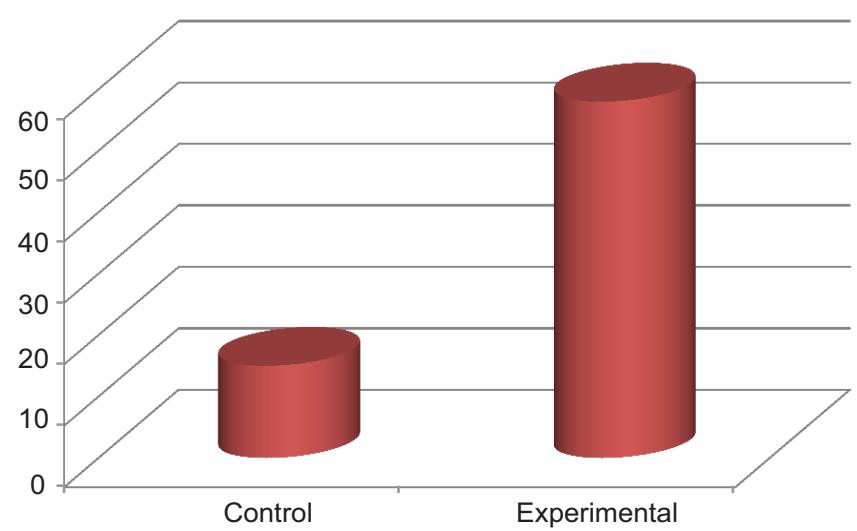

Graph-1: Shows the distribution of data based on types of groups among the study subjects

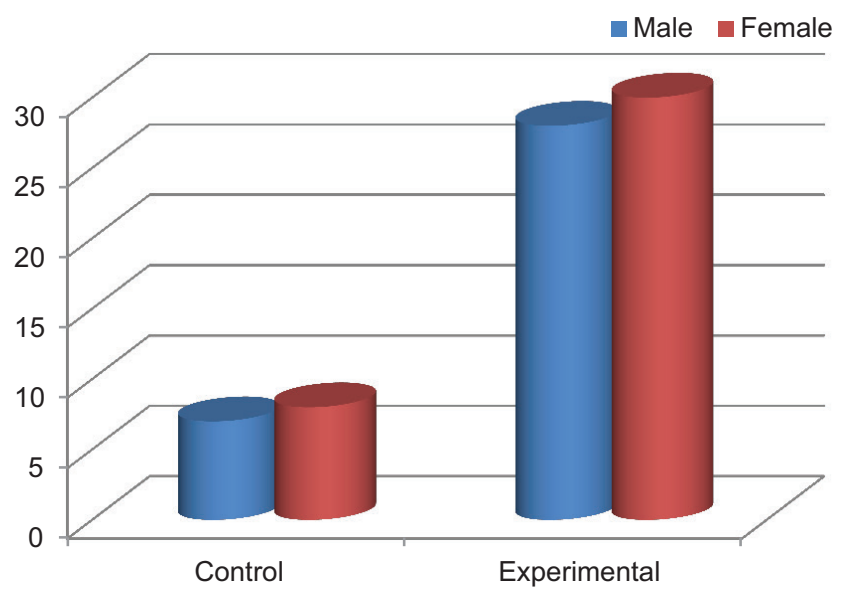

Graph-2: Shows the distribution of data based on gender among control and experimental group between T2 weighted, 3D Fiesta and 3D Cosmic sequences showed significant statistical difference between these three sequences. Significant difference $(\mathrm{p}=0.035)$ between T2 weighted and 3D FIESTA (fast imaging employing steady state acquisition) was noted. Similarly, 3D COSMIC (coherent oscillatory state acquisition for the manipulation of image contrast) and T2 weighted sequence shows significant difference at $\mathrm{p}$ value 0.001 and $3 \mathrm{D}$ COSMIC and 3D FIESTA shows significant difference at $\mathrm{p}$ value 0.009 . The FOV (field of view) was found to be increased in cosmic sequence than Fiesta and 2D. Matrix was found to be $320 * 320$ in cosmic sequence when compared to Fiesta and 2D. Slice thickness was same in Cosmic and Fiesta whereas in $2 \mathrm{D}$ it was about 3. Inter-slice spacing was found to be 0.5 in T2W FSE and none in Fiesta and Cosmic sequences. TR (repetition time) and TE (Echo time) was found to be 5.1 and 2.5 respectively (Table 1,2 and 3 ).

There were 15 control patients and 58 experimental patients. About 58 patients with clinical suspicion of lumbar radiculopathy imaging were enrolled in the study. In control group, about 7 males and 8 females were included whereas 28 males and 30 females were included in experimental group (Graph 1 and 2).

\section{DISCUSSION}

In our study, we were clearly able to visualize the exit of nerves from the cord and cauda equina at various foramina levels with the addition of 3D FIESTA and 3D COSMIC sequences. Majority of our patients were found to have L5S1 Diffuse annular disc bulge with impingement on the traversing nerve roots which was clearly depicted by $3 \mathrm{D}$ FIESTA and 3D COSMIC sequences.

Routine $\mathrm{T} 2 \mathrm{~W}$ sequences with their increased slice thickness had good resolution when compared with 3D sequences. However, the probability of missing the findings was increased here. However, 3D FIESTA and 3D COSMIC sequences with their isotropic image resolution and greater degree of contrast had greater sensitivity in the grading of nerve root involvement in cases of lumbar radiculopathy. ${ }^{9}$

The contrast between the nerve roots, surrounding peripheral tissues and adjacent structures was less in 3D FIESTA sequence in comparison with $3 \mathrm{D}$ COSMIC sequence. 3 D COSMIC sequence shows excellent soft-tissue contrast. Contrast to noise ratio and Signal to noise ratio of lumbar spine tissue was much better in 3D COSMIC sequence. ${ }^{10}$

In the present study, it was found that $3 \mathrm{D}$ sequences in Magnetic Resonance Imaging gives us very good details about morphology because of the usage of thin sections with higher Signal to noise ratio. Also, multiplanar reconstruction helps in viewing from different directions. 3D sequences with their improved contrast resolution aids in better visualization of nerve roots. 3D COSMIC sequence with its pre steadystate imaging technique delivers ideal visualization of soft tissue structures which are adjacent to bony structures. Also, with the addition of fat saturation to $3 \mathrm{D}$ sequences chemical shift artifacts can be overcome.

In a study conducted by Hiroyuki Takashima et al, it was observed that 3D sequences with high Signal to noise ratios (SNRs) are excellent for detecting morphologic changes and 
these findings are in concordance with the findings of the present study. Additionally, SNR was found to be equal to the ratio of the average signal intensity over the standard deviation of the noise and an important reference of MRI. Because of the contrast in the FIESTA, water components and blood appear as high signals, but the soft-tissue signals with short T2 values give lower signal intensity. ${ }^{11,12}$

In a study conducted by Tanitame $\mathrm{K}$ and $\mathrm{Zhang} Z$ et al, they suggested that the FIESTA sequence was seen with lower contrast between the peripheral tissues and the nerve root and its structures, such as bone or intervertebral discs, and it was impossible to distinguish the nerve root whereas the COSMIC was performed before echo sampling reached a steady state and exhibits greater soft-tissue contrast. This provided improvement in the CNR and SNR (Signal-tonoise ratio) of cervical-spine tissue including the spinal cord, vertebral discs, and nerve root canal, as well as the contrast between CSF and nerve roots. ${ }^{13,14}$

When the comparison of COSMIC and COSMIC-FS (fat-suppression) on the intra- and extra-foramen was done, it was seen that the CNR between the nerve root and peripheral tissue was significantly higher for COSMIC-FS than for COSMIC. To distinguish between the nerve root from the intervertebral foramina, COSMIC-FS leads to better contrast between the nerve root and intervertebral disc and bone with improvemennt in the intervertebral foramen and external zone where epidural fat is present, due to the concomitant use of fat suppression made the inhibition of chemical shift artifacts possible. ${ }^{15}$ The results of our study suggested that either sequence is useful for detecting intradural lesions, and that COSMIC is superior for detecting nerve root lesions that are adjacent to soft-tissue inside and outside the intervertebral foramina, where there is little water. Further research is needed with larger sample size to compare the images obtained with FIESTA, COSMIC and $2 \mathrm{D}$ under the same conditions.

\section{CONCLUSION}

The higher signal to noise ratio and contrast to noised ratio in $3 \mathrm{D}$ COSMIC sequence in comparison with $3 \mathrm{D}$ FIESTA and 2D sequences makes it an ideal sequence for detecting even subtle morphologic changes. The higher spatial resolution makes multi planar reconstruction possible without causing much degradation of the image. Hence, 3D sequences are better than $2 \mathrm{D}$ sequences in the imaging of lumbar radiculopathy and also 3D COSMIC definitely has advantages over 3D FIESTA sequence.

\section{REFERENCES}

1. Lou $\mathrm{ZH}, \mathrm{Qu} \mathrm{JR}, \mathrm{Li} \mathrm{HL}$, et al. Optimal technique of three-dimensional MRI of the lumbar nerve root and its radicular vein in normal and lumbar disc herniation patients. Chin Med J (Engl) 2011; 124(1):1802-1806.

2. Bieri O, Scheffler K. Fundamentals of balanced steady state free precession MRI. J Magn Reson Imaging. 2013; 38(5):2-11.

3. Byun WM, Ahn SH, Ahn MW. Value of 3D MR lumbosacral radiculography in the diagnosis of symptomatic chemical radiculitis. AJNR Am J Neuroradiol. 2012; 33(2):529-534.
4. Byun WM, Jang HW, Kim SW. Three-dimensional magnetic resonance rendering imaging of lumbosacral radiculography in the diagnosis of symptomatic extraforaminal disc herniation with or without foraminal extension. Spine (Phila Pa 1976) 2012; 37(4):840-844.

5. Jarvik J, Deyo R. Diagnostic evaluation of low back pain with emphasis on imaging. Ann Intern Med. 2002; 137(6): 586-595.

6. Ludwinski FE, Gnanalingham K, Richardson SM, Hoyland JA. Understanding the native nucleus pulposus cell phenotype has important implications for intervertebral disc regeneration strategies. Regen Med 2013; 1(5):75-87.

7. Melhem ER. Technical challenges in MR imaging of the cervical spine and cord. Magn Reson Imaging Clin N Am 2000; 8(4):435-452.

8. Boden SD, Davis DO, Dina TS, et al. Abnormal magnetic-resonance scans of the lumbar spine in asymptomatic subjects. J Bone Joint Surg Am. 1990; 72(2):403-8.

9. Lichy MP, Wietek BM, Mugler JP 3rd, Horger W, Menzel MI. Magnetic resonance imaging of the body trunk using a single-slab, 3-dimensional, T2-weighted turbo-spin echo sequence with high sampling efficiency (SPACE) for high spatial resolution imaging: initial clinical experiences. Invest Radiol 2005; 40(6):754-760.

10. Shishido H, Takashima $H$, Takebayashi $T$, et al. Visualization of the foramen intervertebral nerve root of cervical spine with 3.0 tesla magnetic resonance imaging: a comparison of three-dimensional acquisition techniques. Nihon Hoshasen Gijutsu Gakkai Zasshi 2014; 70(1):670-5.

11. Hiroyuki Takashima, Tsuneo Takebayashi, Hiroki Shishido, Mitsunori Yoshimoto, Rui Imamura, Yoshihiro Akatsuka, Yoshinori Terashima, Hiroyoshi Fujiwara, Masateru Nagae, Toshikazu Kubo and Toshihiko Yamashita. Comparison with Magnetic Resonance Three-Dimensional Sequence for Lumbar Nerve Root with Intervertebral Foramen. Asian Spine Journal 2016; 1(3): 59-64.

12. Shen J, Wang HY, Chen JY, Liang BL. Morphologic analysis of normal human lumbar dorsal root ganglion by 3D MR imaging. AJNR Am J Neuroradiol 2006; 27(5):2098-103.

13. Tanitame K, Tanitame N, Tani C, et al. Evaluation of lumber nerve root compression using thin-slice thickness coronal magnetic resonance imaging: threedimensional fat-suppressed multi-shot balanced nonsteady-state free precession versus three-dimensional T1-weighted spoiled gradient-recalled echo. Jpn J Radiol. 2011; 29(4):623-629.

14. Zhang Z, Song L, Meng Q et al. Morphological analysis in patients with sciatica: a magnetic resonance imaging study using three-dimensional high-resolution diffusion-weighted magnetic resonance neurography techniques. Spine (Phila Pa 1976) 2009; 34(2):E245-50.

15. Haacke EM, Wielopolski PA, Tkach JA, Modic MT. Steady-state free precession imaging in the presence of motion: application for improved visualization of the cerebrospinal fluid. Radiology 1990; 175(1):545-52.

Source of Support: Nil; Conflict of Interest: None

Submitted: 28-02-2019; Accepted: 26-03-2019; Published online: 30-10-2019 